day discharge. The MRI result changed management in $88 \%$ of patients.

Conclusion A significant proportion of patients experienced delay to discharge due to delay of inpatient MRI. The result of MRI changed treatment plans for most patients, and allowed same day discharge in almost one third of cases. Timely access to inpatient MRI is a critical and potentially modifiable variable that may reduce length of stay and expedite treatment for neurology inpatients.

\section{A CASE REPORT OF MYELIN OLIGODENDROCYTE GLYCOPROTEIN ANTIBODY POSITIVE ENCEPHALITIS MIMICKING HANDL SYNDROME WITH SUBSEQUENT ACUTE OPTIC NEURITIS}

${ }^{1}$ Christopher Belder, ${ }^{2}$ Thomas Kimber. ${ }^{1}$ Neurology, Flinders Medical Centre, Adelaide, SA, Australia; ${ }^{2}$ Neurology, Royal Adelaide Hospital, Adelaide, SA, Australia

\subsection{6/bmjno-2021-ANZAN.60}

Background A 22 year-old right-handed female presented with acute onset of speech disturbance. There was no visual/ motor deficit or clinical seizure activity. CT brain/angiography/perfusion demonstrated hypoperfusion in the left temporo-parieto-occipital region (not conforming to a single vascular territory).

There was subsequent improvement in her speech, and she reported a severe left temporal headache with associated nausea and photophobia. An urgent MRI brain was normal. She was diagnosed with migraine with dysphasic aura and treated symptomatically with ongoing improvement. On review the next day she reported mild ongoing headache, but her neurological examination was normal with no dysphasia.

2 days later, she developed right retro-orbital pain, which was followed a further 3 days later by right eye monocular visual impairment. Clinical examination was consistent with acute right optic neuritis. Repeat MRI brain/orbits demonstrated new changes of right optic neuritis with perineuritis and a left thalamic T2-hyperintense lesion. CSF studies demonstrated a monocytic pleocytosis.

The clinical impression was of possible myelin oligodendrocyte glycoprotein (MOG) antibody disease leading to optic neuritis and unilateral cortical encephalitis, manifesting as cortical spreading depression/migraine aura.

She was commenced on IV methylprednisolone followed by an oral prednisolone taper with complete symptom resolution.

Her serum subsequently tested positive for MOG antibody. Conclusions MOG-antibody disease can cause a unilateral encephalitis that may cause attacks of cortical spreading depression. This case demonstrates that this may occur in the absence of the previously reported significant unilateral cortical MRI changes - we hypothesise that some patients previously diagnosed with 'HANDL syndrome' may have MOGantibody disease.

\section{REFERENCES}

1. Ogawa R, Nakashima I, Takahashi T, et al. MOG antibody-positive, benign, unilateral, cerebral cortical encephalitis with epilepsy. Neurol Neuroimmunol Neuroinflamm 2017:4(2):e322. Published 2017 Jan 16. doi:10.1212/ NXI.0000000000000322

2. Matoba, S, Inoue, M, Morihata, H, Takeshima, T. Case report of myelin oligodendrocyte glycoprotein antibody-positive encephalitis mimicking hemiplegic migraine. Neurol Clin Neurosci 2020;8:323- 325. https://doi.org/10.1111/ncn3.12420

\section{1 \\ FEASIBILITY OF INSTITUTING GRADUATED HIGH INTENSITY TRAINING FOR PARKINSON DISEASE (FIGHT- PD); A NON-CONTACT BOXING EXERCISE STUDY}

${ }^{1}$ David J Blacker, ${ }^{2}$ Travis Cruickshank, ${ }^{2}$ Mitchel Turner, ${ }^{1}$ Claire Tucak, ${ }^{1}$ Rai Fazio. ${ }^{1}$ Perron Institute for neurological and translational science, Nedlands, WA, Australia; ${ }^{2}$ Exercise Physiology, Edith Cowan University, Joondalup, WA, Australia

\subsection{6/bmjno-2021-ANZAN.61}

Objectives Preliminary evidence suggests non-contact boxing exercise is feasible and possibly beneficial for Parkinson Disease (PD). Current studies lack detailed description of component elements and documentation of exercise intensity. We present the protocol of FIGHT-PD; which explores the feasibility, tolerability and safety of a non-contact boxing exercise program for PD, developed by a neurologist (who has PD), a professional boxing trainer, a neurophysiotherapist and exercise physiologists.

Methods Twenty early stage (Hoehn and Yahr 1 and 2) PD subjects will undergo baseline evaluations of $\mathrm{PD}$ and cardiac stress testing. Training includes quantifiable balance and movement drills, high intensity aerobic bursts, and sequences of punches using the Fightmaster training machine.

Over 15 weeks, three 30-60 minute workouts per week will be conducted in three, 4 week blocks separated by rest weeks. Block one focuses on technique; the second escalates the physical intensity, and the third adds cognitive challenges. Rate of perceived physical exertion (RPE) and mental exertion will be measured by the Borg scale for every component of each workout, and heart rate continuously recorded by Polar monitors. Numerous standardised PD scales and a body chart discomfort scale will be administered at each workout, monitoring the development of pain or injuries. These observations will provide the primary outcomes of tolerability and safety, and secondary outcomes of quantified heart rate measuring exercise intensity, and effect on quality of life. Feasibility details including recruitment, retention and adherence rates will be measured.

Conclusions This trial should provide essential details to plan future exercise-based studies.

\section{PEMBROLIZUMAB INDUCED LAMBERT-EATON MYASTHENIC SYNDROME}

${ }^{1}$ Jasmine Ashhurst, ${ }^{2}$ Rami Haddad, ${ }^{3}$ Rob Zielinski. 'St George Hospital, Sydney, NSW, Australia; ${ }^{2}$ Neurology, Orange Health Service, Orange, NSW, Australia; ${ }^{3}$ Medical Oncology, Orange Health Service, Orange, NSW, Australia

\subsection{6/bmjno-2021-ANZAN.62}

Case Report Lambert-Eaton Myasthenic Syndrome (LEMS) is a neuromuscular disorder caused by antibodies directed to the presynaptic voltage-gated calcium channel. It is often paraneoplastic, most commonly associated with Small Cell Lung Cancer (SCLC).

This report outlines the case of a patient who developed LEMS secondary to pembrolizumab treatment for metastatic melanoma.

An 82 year-old female presented to hospital 1 week after cycle 2 of pembrolizumab treatment for metastatic melanoma.

On examination, she was found to have dysphagia, ocular muscle weakness and generalised weakness (most markedly weakness in hip flexors). Her weakness was fatigable and she 
had a waddling gait. Clinical picture was consistent with a clinical diagnosis of LEMS rather than myositis, which was confirmed by elevated anti-VGCC antibodies and response to Acetylcholinesterase inhibitors.

Results Anti-VGCC antibodies elevated at $119 \mathrm{pM}(<30)$.

Transiently elevated CK, negative myositis autoantibodies, negative anti-MuSK antibodies, negative AChR antibodies.

Although repetitive nerve stimulation did not show increment in the right ulnar CMAP after isometric muscle activation, the clinical picture was consistent with LEMS.

Marked improvement to treatment with oral prednisone and pyridostigmine. Due to side effects, pyridostigmine was changed to 3,4-Diaminopyridine therapy with excellent response.

Steroids were weaned off and the patient is adequately controlled on 3,4-Diaminopyridine.

Conclusion Our case report shows that LEMS can arise as a result of an immune-related adverse event (irAE) to pembrolizumab; an Anti-PD-1 Monoclonal Antibody. The immune response persists after cessation of this checkpoint inhibitor medication. It is important to recognise and treat this condition early.

\section{3 'HAVE I GONE MAD?': A CASE OF ALICE IN WONDERLAND SYNDROME}

${ }^{1}$ Jasmine Ashhurst, ${ }^{2}$ Rami Haddad, ${ }^{3}$ Matthew Tait. 'St George Hospital, Sydney, NSW, Australia; ${ }^{2}$ Neurology, Orange Health Service, Orange, NSW, Australia; ${ }^{3}$ Neurosurgery, Nepean Hospital, Sydney, NSW, Australia

\subsection{6/bmjno-2021-ANZAN.63}

\section{Case Report}

Introduction Alice in Wonderland Syndrome (AIWS) is a rare neurological disorder that is characterised by unusual distortions to perception. The aetiology of AIWS is unclear, though it has been reported in a number of neurological conditions including infection (esp. EBV), CNS lesions, migraines and as a side effect to medications.

This report outlines the case of a 72 year old gentleman who presented to hospital due to visual changes which he described as dysmetropsia (objects appeared distorted - thinner) and episodic chromatopsia. His chromatopsia was described as his entire visual field coloured with an orange hue, though this colour changed throughout the course of his admission. Movements appeared to occur in slow motion and movements of humans had a robotic appearance. Neurological examination was otherwise normal.

These symptoms resolved after 2 days, and on subsequent examination his neurological and ophthalmological examinations were unremarkable.

Results MRI brain revealed a right occipito-temporal T2 hyperintense lesion, initially interpreted as a subacute ischaemic infarct. Repeat MRI with contrast revealed a stable appearance of the lesion with areas of subtle contrast enhancement. PET scan showed reduced metabolic activity within the lesion with reduced FDG accumulation.

Biopsy of the lesion identified features of a diffuse astrocytoma.

Conclusions AIWS is a poorly recognised syndrome. Symptoms are not typical for an ischaemic event and alternative diagnosis should be investigated as an explanation for the cause of visual distortions.

\section{FALSE POSITIVE RT-QUIC TEST FOR CREUTZFELDT JAKOB DISEASE IN DEMENTIA WITH STATUS EPILEPTICUS}

${ }^{1}$ Rajiv Wijesinghe, ${ }^{2}$ Craig Anderson, ${ }^{2}$ Miriam Welgampola, ${ }^{3}$ Christine Stehmann, ${ }^{3}$ Steven Collins, ${ }^{1}$ Penelope Spring. 'Department of Neurology, Concord Repatriation General Hospital, Concord, NSW, Australia; ${ }^{2}$ Department of Neurology, Royal Prince Alfred Hospital, Camperdown, NSW, Australia; ${ }^{3}$ Australian National CJD Diagnostic Service, Florey Institute for Neuroscience and Mental Health, Parkville, VIC, Australia

\subsection{6/bmjno-2021-ANZAN.64}

We present the case of a 72-year-old woman with likely false positive tests for Creutzfeldt Jakob disease (CJD). She had a background of focal epilepsy and dementia, related to previous alcohol abuse and head trauma. On her initial presentation in October 2019, she was drowsy with continuous left sided focal motor seizures. An EEG demonstrated continuous periodic lateralised epileptiform discharges (PLEDs) arising from the right temporal region, consistent with status epilepticus. She was commenced on levetiracetam and subsequently lacosamide, with seizure resolution over 12 days. Despite control of her seizures, she remained significantly cognitively impaired. A cerebral MRI demonstrated asymmetric cortical and thalamic diffusion restriction and the possibility of sporadic CJD was raised. A lumbar puncture revealed a normal total protein $(0.4 \mathrm{~g} / \mathrm{L})$, a positive 14-3-3 protein but an undetectable tau protein. She was eventually discharged to a residential aged care facility, however returned to a different hospital in January 2020, obtunded with recurrent generalized seizures. Further results of her previous CSF examination were now available, revealing a positive RT-QuIC assay. However, a repeat MRI brain demonstrated resolution of the previous regions of diffusion restriction. Serial EEGs demonstrated continuous right temporal PLEDs which improved after the addition of sodium valproate. Repeat cognitive screening was markedly improved, however not quite reaching her 2019 baseline. We suggest that a false positive RT-QuIC test probably arose from status epilepticus in the context of significant pre-existing cerebral pathology. Excluding a pre-mortem diagnosis of CJD may be challenging in the setting of recurrent seizures.

\section{EBV AND NMDA RECEPTOR ANTIBODY POSITIVE OPSOCLONUS-MYOCLONUS SYNDROME IN AN IMMUNOCOMPROMISED PATIENT WITH RENAL CLEAR CELL CARCINOMA: A CASE REPORT}

${ }^{1}$ Catherine Ashton, ${ }^{2}$ Janavi Dunuwille. ${ }^{1}$ Neurology Department, Fiona Stanley Hospital, Murdoch, WA, Australia; '2Department of neurology, Sir Charles Gairdner Hospital, Nedlands, WA, Australia

\subsection{6/bmino-2021-ANZAN.65}

Introduction Rare cases of double positive Epstein-Barr virus (EBV) and anti-N-methyl-D-aspartate receptor (anti-NMDAR) antibody causing encephalitis have been described associated with solid organ transplant. ${ }^{1-4}$ Opsoclonus-myoclonus syndrome is often a paraneoplastic or parainfectious phenomenon, but has only rarely been described associated with antiNMDAR and usually with additional neuropsychiatric symptoms or encephalopathy. ${ }^{5-6}$ We describe the rare case of a renal transplant patient with opsoclonus-myoclonus syndrome 